\title{
INVESTIGACIONES
}

\section{Praxis y herramientas del psicólogo/a en equipos psicosociales y visita domiciliaria escolar: Nuevos desafíos en educación*}

\author{
Praxis and tools of the psychologist in psychosocial teams and school's home visits: \\ New challenges in education
}

\author{
Constanza Pedreros Carrasco ${ }^{a}$, Marcela Aracena Álvarez ${ }^{b}$ \\ ${ }^{a}$ Fundación Arturo López Pérez. \\ capedreros@uc.cl \\ ${ }^{b}$ Escuela de Psicología, Facultad de Ciencias Sociales, Pontificia Universidad Católica de Chile. \\ maracena@uc.cl
}

\section{RESUMEN}

A partir de las reformas educacionales en Chile las escuelas se ven enfrentadas a nuevos desafíos en convivencia escolar y al abordaje integral del estudiante. Algunas escuelas incorporan Equipos Psicosociales (EP) para adoptar este nuevo enfoque, siendo apoyadas por fondos gubernamentales y municipales. En Chile la información sobre EP en contexto escolar es escasa, por lo que surge el interés de dar una primera aproximación sobre las prácticas y rol que realiza el EP en la escuela y la utilización de la Visita Domiciliaria Escolar (VDE). Mediante un diseño de investigación cualitativa de tipo descriptivo, se realizaron seis entrevistas semiestructuradas y un debate grupal. Participaron once psicólogos/as y trabajadores/as sociales de la comuna de San Joaquín, Santiago de Chile. Para el análisis se utilizó codificación temática. Finalmente, los resultados muestran las potencialidades y desafíos del EP y VDE para el bienestar integral infantil.

Palabras claves: Intervención psicosocial, bienestar integral, convivencia escolar.

\begin{abstract}
Schools are faced with new challenges in school life and comprehensive approach to the student from the educational reforms in Chile. Some schools incorporate Psychosocial Teams (PT) to adopt this new approach, being supported by governmental and municipal funds. In Chile, information on PT in a school context is limited, so there is an interest to give a first approximation of the practices and role of the PT in the school and the use of the School's Home Visit (SHV). Through a qualitative research design of descriptive type, six semi structured interviews and a group discussion were elaborated. Eleven psychologists and social workers from San Joaquin district, Santiago of Chile participated in this study. For the analysis, thematic coding was used. Finally, the results show the potentialities and challenges of PT and SHV for the integral well-being of children.
\end{abstract}

Key words: psychosocial intervention, integral wellbeing, school life.

\footnotetext{
* Investigación financiada por el proyecto FONDECYT N 11140679 "La visita domiciliaria como estrategia de intervención social para aumentar la participación de los padres en la educación de sus hijos en escuelas básicas municipales".
} 
Estudios Pedagógicos XLVII N² 2: 117-136, 2021

PRAXIS Y HERRAMIENTAS DEL PSICÓLOGO/A EN EQUIPOS PSICOSOCIALES Y VISITA DOMICILIARIA ESCOLAR: NUEVOS DESAFÍOS EN EDUCACIÓN

\section{INTRODUCCIÓN}

\subsection{ESCUELA Y BIENESTAR INTEGRAL INFANTIL}

La Ley General de Educación en Chile define educación como un proceso que tiene por finalidad alcanzar el desarrollo espiritual, ético, moral, afectivo, intelectual, artístico y físico (Ley n ${ }^{\circ}$ 20.370, 2009). Desde esta nueva comprensión, las escuelas tienen la responsabilidad de brindar al estudiante un medio saludable donde pueda desarrollarse íntegramente de modo de facilitar los procesos educativos (Ley no 20.370, 2009), más aún en poblaciones de alto riesgo.

A partir del Plan de Mejoramiento Educativo (PME), se integran conceptos de bienestar psicosocial y desarrollo integral del estudiante reconociéndolos como parte de los derechos de los niños/as y jóvenes (Larraín, 2011). Sin embargo, hasta este momento en Chile no hay ninguna reforma que entregue lineamientos sobre el uso de recursos, diseño o evaluación de intervenciones psicosociales, lo cual dificulta su aplicación en escuelas (Gatica \& Alfaro, 2015). Dado que no se ha establecido homogeneidad, no existen indicadores nacionales del trabajo psicosocial, por lo que no existe evidencia que asegure que actualmente los recursos sean invertidos costo-efectivamente, ni tampoco que las intervenciones utilizadas sean las más adecuadas para el contexto chileno (Gatica \& Alfaro, 2015).

Las reformas coinciden con la información encontrada en literatura, donde se ha reportado que el rendimiento académico es afectado por diversos elementos contextuales y personales del estudiante (Arévalo \& Santana, 2013). Los indicadores de riesgo psicosocial se han asociado con deserción escolar, fracaso académico, problemáticas de salud pública, salud mental, convivencia escolar, bienestar global, entre otros (Jadue, 2002; Gavilán, Quiles \& Chá, 2005; Leal-Soto, 2005; Cornejo \& Redondo, 2007; Yager \& Walton, 2011; Walsh, Lee-St. John, Raczek \& Foley, 2015). En la revisión publicada por Yager y Walton, (2011), se analiza la efectividad de varias intervenciones psicosociales en escuela, pero se concluye que, a pesar de la evidencia empírica, todavía hay desconocimiento en el cuerpo académico del efecto que tienen las intervenciones psicosociales. Según Gavilán et al. (2005) en escuelas existe la necesidad, pero debido al desconocimiento de los beneficios de intervenir en el área psicosocial, no se han aplicado medidas al respecto y los problemas persisten.

En la actualidad, el Estado de Chile invierte recursos para intervenir convivencia escolar en escuelas que presenten alto riesgo social mediante la Ley de Subvención Escolar Preferencial (SEP) No 20.248 (2008). Desde esta nueva perspectiva nace la necesidad de incorporar nuevas profesiones desde las Ciencias Sociales al ámbito educativo (Gatica \& Alfaro, 2015) para asegurar la calidad de la educación de los niños y niñas en contextos de vulnerabilidad social mediante el mejoramiento de las condiciones del ambiente escolar (Ley n $\left.{ }^{\circ} 20.248,2008\right)$.

\subsection{EQUIPO O DUPLA PSICOSOCIAL}

Para cumplir con los nuevos requerimientos legales, algunas escuelas han optado por contratar duplas o equipos psicosociales. La definición de Dupla Psicosocial es variable, según el contexto donde se utiliza y al perfil profesional de quienes la componen (Ministerio de Salud de Chile [MINSAL], 2008; Aracena et al., 2011). Surge desde la intervención comunitaria como una estrategia para complementar las intervenciones sociales, aunque rápidamente 
se extendió a otras disciplinas (Santana \& Farkas, 2007; Gómez \& Haz, 2008; López et al., 2011; Berroeta, Hatibovic \& Asún, 2012). A pesar de su popularidad, en la literatura no se encontró información sobre la efectividad de las duplas en contexto educativo.

A nivel internacional se ha reportado la efectividad de las intervenciones psicosociales, aplicadas por distintos profesionales, para favorecer procesos educativos (Jadue, 2002; Gavilán et al., 2005; Leal-Soto, 2005; Cornejo \& Redondo, 2007; Gastañaga, 2007; Yager \& Walton, 2011; Walsh et al., 2014; Walsh et al., 2015). Por otro lado, estudios internacionales han demostrado que las intervenciones realizadas por psicólogos/as y trabajadores/as social, individualmente, han obtenido resultados favorables en resultados académicos e indicadores de bienestar escolar (Allen \& Tracy, 2004; Franklin, Kim \& Tripodi, 2009; García \& Cohen, 2010; Vásquez, 2015). Sin embargo, en la revisión de la literatura internacional utilizando los motores de búsqueda Scielo, NCBI, ERIC, no se encontró evidencia sobre intervenciones realizadas por Duplas Psicosociales en escuelas. Respecto a la evidencia en Chile se reportan dos investigaciones relevantes: un proyecto del Fondo Nacional de Ciencias y Tecnología (FONDECYT) liderado por Saracostti (2013) y un estudio de Gatica y Alfaro (2015) en donde se analiza el funcionamiento de los recursos SEP. A continuación, se exponen algunos de los resultados de ambas investigaciones.

Para fines de esta investigación, se entiende Dupla o Equipo Psicosocial (DP/EP) como el trabajo en equipo de psicólogos/as y trabajadores/as sociales, para abordar problemáticas del tipo psicosocial que afectan al estudiante en la escuela para su pronta detección, derivación y/o tratamiento (Arévalo \& Santana, 2013). Respecto a las actividades principales de la dupla se destacan: el contacto con las familias, entrevistas, intervenciones psicosociales $^{1}$, visita domiciliaria, análisis de casos de intervención, trabajo de promoción y prevención, además de intervenir en el clima laboral y brindar herramientas a profesores y directivos para el trabajo con niños, entre otras (Saracostti \& Santana, 2013).

Sobre los desafíos del trabajo psicosocial, distintos estudios internacionales coinciden en que, a pesar de la evidencia empírica sobre la relevancia de las intervenciones psicosociales en los procesos educativos, todavía hay desconocimiento por parte de profesores y directivos, por lo que no son implementadas (Gavilán et al., 2005; Yager \& Walton, 2011). En el caso de Chile, Gatica y Alfaro (2015) evidencian que el rol y actividades del equipo psicosocial en escuelas no está delimitado, generando confusión en el ejercicio del trabajo para el profesional y la escuela, perjudicando la calidad, continuidad y evaluación de las intervenciones.

\subsection{VISITA DOMICILIARIA}

Dentro de las intervenciones que realizan los EP se destaca la Visita Domiciliaria. Esta se define como "la entrega de un servicio de ayuda, información o guía a un individuo desde el embarazo hasta la adultez mayor y/o a una familia, que puede ir desde la prevención hasta la intervención y evaluación." (Razeto, 2016, p. 457).

Tanto a nivel internacional como nacional existe validación empírica sobre su efectividad en programas para protección de la infancia, tales como Early Head Start (pionero en vincular salud y educación con éxito en Estados Unidos), Sure Start (Inglaterra),

Intervención psicosocial se entiende como el trabajo integral sistémico, con foco familiar, social, psicológico, emocional, sustentado en el apoyo en red (Saracostti \& Santana, 2013). 
Project Elmira (EE.UU.), entre otras (MINSAL, 2008; Schodt, Parr, Araujo \& RubioCodina, 2015; Office of Planning, Research and Evaluation [OPRE], 2016). Un ejemplo de Centroamérica es el programa cubano "Educa tu Hijo", el cual ha tenido éxito en indicadores de desarrollo infantil y habilidades parentales (Siverio, 2011). Sin embargo, solo unos pocos programas se extienden hasta infancia media, que es la población objetivo de este trabajo, por lo que el impacto de la visita en esta población no es claro (OPRE, 2016).

Específicamente la Visita Domiciliaria Escolar (VDE), es utilizada para la observación y comprensión del entorno del niño/a (condiciones físicas y dinámica del hogar), detectar las necesidades educativas, fortalecer el vínculo escuela-hogar y lograr entregar servicios a familias aisladas física y/o socialmente (Allen \& Tracy 2004; Meyer, Mann \& Becker, 2011). Sin embargo, la evidencia nacional e internacional sobre su impacto en el contexto educativo es escasa (Razeto, 2016).

A nivel internacional, se encuentran programas de VDE realizadas por profesores para mejorar el vínculo familia-escuela y procesos de aprendizaje (Meyer et al., 2011). Un ejemplo es “The Home Visit Project" en Sacramento, California, EE.UU., el cual tuvo éxito fortaleciendo el vínculo familia-escuela, aumentando la participación parental en el proceso educativo y mejorando el rendimiento académico (Cowan, Bobby, St. Roseman \& Echandía, 2002).

En Chile no se encuentran estudios que evalúen la VDE, sin embargo, dado el impacto observado en otras disciplinas, Razeto (2016) propone esta herramienta para vincular el espacio educativo con la familia, esto con el fin de abordar problemáticas psicosociales que pudiesen estar afectando la calidad de la educación. Esta hipótesis se sustenta en la propuesta de revitalización de la visita domiciliaria en escuelas como herramienta efectiva para vincular a la escuela con redes (Wasik \& Bryant, 2001; Allen \& Tracy, 2004; Avellar \& Supplee, 2013; Razeto, 2016), desde el modelo ecológico para asegurar el bienestar biopsicosocial infantil (Brofenbrenner, 1986).

A modo de síntesis, a partir de los antecedentes presentados se detecta la necesidad de levantar información respecto a la implementación de los equipos psicosociales y prácticas en visita domiciliara como estrategia psicosocial en el contexto escolar. Por consiguiente, en la presente investigación interesa conocer qué, cómo y con qué fin se realizan intervenciones psicosociales en escuela, cuál es el rol de los equipos psicosociales y psicólogo/a escolar y el uso específico que se le da a la visita domiciliaria escolar. Esta información es fundamental para guiar el diseño de mediciones y propuestas de lineamientos de las intervenciones psicosociales en contexto educativo. Por lo que el presente estudio pretende ser una de las primeras aproximaciones al problema.

\section{METODOLOGÍA}

\subsection{DISEÑO Y OBJETIVOS}

El presente estudio utiliza un diseño carácter cualitativo del tipo descriptivo analítico exploratorio. Desde la mirada de los entrevistados la investigación tiene por objetivo:

a. Identificar y analizar las prácticas realizadas por el Equipo Psicosocial en la escuela.

b. Identificar y analizar las prácticas realizadas por el Equipo Psicosocial durante la Visita Domiciliaria. 
c. Indagar en el(los) rol(es) actual(es) que cumple el Equipo Psicosocial y Psicólogo/a en contexto escolar y Visita domiciliaria.

\subsection{PARTICIPANTES}

Los participantes fueron once Psicólogos/as y Trabajadores/as Sociales quienes trabajan en Escuelas Municipales de la comuna de San Joaquín, Santiago, Chile.

Los criterios de inclusión utilizados fueron:(1) ser Psicólogo/a o Trabajador/a Social, (2) intervenir en convivencia escolar en Escuelas Municipales, (3) ser parte de un Equipo Psicosocial y (4) al menos un miembro de la dupla entrevistada debe tener experiencia en visita domiciliaria mínimo por 5 meses (periodo correspondiente a un semestre académico escolar)2.

Del universo de profesionales que trabajan en dupla, se invitó a participar a todos los profesionales que cumplían con los criterios de inclusión. La muestra final fue compuesta predominantemente por profesionales de sexo femenino (80\%). Sobre el perfil de especialización de los participantes, 7 de 11 tienen capacitación en educación. Un entrevistado declara tener menos de 5 meses de experiencia en visita domiciliaria y dos no realizan visitas (Tabla 1).

Tabla 1. Caracterización de la muestra

\begin{tabular}{|l|l|l|l|}
\hline Género & Profesión & $\begin{array}{c}\text { Experiencia } \\
\text { en VDE }\end{array}$ & \multicolumn{1}{|c|}{ Capacitaciones } \\
\hline Masculino & TS & 10 meses & Mención en Psicología Psicoanalítica. \\
\hline Femenino & PS & 7 meses & Diplomado Estrategias de Comunicaciones Educativas. \\
\hline Femenino & PS & 4 años & $\begin{array}{l}\text { Diplomado en convivencia escolar; Diplomado en sexualidad } \\
\text { y terapia de pareja; Diplomado en evaluación y técnicas } \\
\text { infanto-juvenil. }\end{array}$ \\
\hline Femenino & TS & 1 año 3 meses & $\begin{array}{l}\text { Capacitación Escala de Evaluación Familiar de Carolina del } \\
\text { Norte (NCFAS). }\end{array}$ \\
\hline Femenino & TS & 4 meses & Diplomado en Sistemas de Protección Social. \\
\hline Masculino & PS & 4 años & $\begin{array}{l}\text { Diplomado en sexualidad; Capacitación Ley SEP; Diplomado } \\
\text { en convivencia escolar. }\end{array}$ \\
\hline Femenino & TS & 1 año 5 meses & Sí relacionados a educación, pero sin certificación. \\
\hline Femenino & TS & 11 meses & No \\
\hline Femenino & PS & No realiza & Diplomado en psicología educacional. \\
\hline Femenino & PS & No realiza & $\begin{array}{l}\text { Capacitación en convivencia escolar; Pedagogía de la Convi- } \\
\text { vencia, Capacitación; Gestión de la Convivencia y Ley SEP. }\end{array}$ \\
\hline Femenino & TS & 5 años 4 meses. & $\begin{array}{l}\text { Diplomado Convivencia Escolar; Curso Pobreza, Autonomía } \\
\text { Económica y Género FOSIS; Magíster en Gobierno y Socie- } \\
\text { plomadomado Gerencia Pública con Enfoque de Género; Di- } \\
\text { plomilia, Realidad y Derecho, Mención en Mediación. }\end{array}$ \\
\hline
\end{tabular}

Fuente: elaboración propia. TS: Trabajador/a Social; PS: Psicólogo/a.

2 Este último criterio se establece considerando la alta rotación de personal de las escuelas municipales. 
La elección de la comuna para el estudio se fundamenta principalmente en que San Joaquín representa un escenario desafiante para la actividad de las escuelas, esto debido a los altos índices de vulnerabilidad observados en barrios emblemáticos de la comuna. Tomando el ejemplo del barrio de La Legua, el 74\% de los hogares en el sector se clasifica en el primer quintil de mayor vulnerabilidad social (según su puntaje en la Ficha de protección social nacional), el 98,9\% de los hogares presentan hacinamiento y el 22,5\% de los detenidos de la comuna residen en este barrio (Fundación Paz Ciudadana, 2017). En encuestas realizadas a los vecinos de La Legua por la Fundación Paz Ciudadana (2018) muestran que "Las situaciones que generan mayor inseguridad en los vecinos La Legua son las balaceras, el enfrentamiento armado entre bandas o personas, la venta/consumo de drogas, y potenciales asaltos en las calles" (p. 7).

Estos indicadores contextuales influyen altamente en el desarrollo los niños/as (Brofenbrenner, 1986), aumentando el interés y relevancia por conocer los recursos que tienen las escuelas para enfrentar la realidad que afecta a sus estudiantes, y hasta qué punto estas medidas son efectivas.

\subsection{TÉCNICAS DE PRODUCCIÓN DE DATOS}

Para la producción de datos se utilizan entrevistas semiestructuradas (individuales y en dupla) y un debate grupal. El complemento de ambos instrumentos permite aumentar la rigurosidad del estudio (Martínez, 2006). El debate grupal permitió ampliar la mirada, mostrando actitudes y opiniones emergentes en contexto de socialización, además de regular opiniones extremas debido a la negociación entre los mismos participantes (Flick, 2012). Esta técnica también facilitó nuevas reflexiones, debates y resolución de problemas en torno a procesos, situaciones o desafíos presentes en la realidad en común (Flick, 2012). La información se registró mediante grabaciones de audio digital, previa autorización de los participantes, por lo que el tipo de dato corresponde a formato cualitativo de estructura compleja, origen primario y con énfasis en el verbatum. Las grabaciones se extienden en promedio por una hora.

\subsection{PROCEDIMIENTO Y ANÁLISIS DE DATOS}

El medio de contacto con los participantes fue a través de un informante clave perteneciente a la Dirección de Educación de la Municipalidad de San Joaquín, quien los invitó a participar vía correo electrónico. Del total de ocho escuelas municipales, dos declinan la invitación. Las entrevistas se realizaron desde diciembre del 2016 a marzo del 2017 y el debate grupal se realizó en junio del 2017.

Para las entrevistas se elaboró una pauta con preguntas sobre el rol y actividades que realiza el Equipo Psicosocial y psicólogo/a, descripción de la visita domiciliaria, apoyo institucional a las intervenciones psicosociales. Las entrevistas se realizaron en los lugares de trabajo de los participantes. Posteriormente, se evaluó los campos temáticos a profundizar y se elaboró una segunda pauta para el debate grupal. Los temas abordados en el debate grupal fueron los desafíos de implementación de la dupla en la escuela y prácticas de autocuidado. Participaron cuatro integrantes de las Duplas ya entrevistadas.

Para el análisis se utiliza codificación temática, la cual consiste en el desarrollo de una teoría a partir de la distribución de perspectivas sobre un proceso (Flick, 2012). Comparte las 
bases de la Grounded Theory, pero se diferencia en que parte por el análisis de casos, para luego definir una estructura temática (realizada a partir de las categorías relevantes de los casos) para luego hacer una comparación cruzada entre datos de entrevistas y debate grupal (Flick, 2012). Para el presente estudio se analizaron 6 entrevistas y una discusión grupal mediante una codificación abierta de las transcripciones a través del software para análisis de datos cualitativos Atlas ti., con el fin de extraer conceptos e ideas centrales emergentes. Posteriormente se generó la estructura temática la cual utilizó para elaborar la pauta temática para el debate grupal. Luego se realizó una transcripción, categorización y subcategorización de ambos instrumentos, facilitando la organización definitiva de los resultados.

Como unidades de análisis se consideraron las secuencias discursivas de carácter descriptivo, argumentativo o explicativo que se refieren al rol y actividad del profesional que interviene en escuela, definiendo como ejes temáticos: rol y actividades que realiza el psicólogo/a y equipo psicosocial, desafíos del trabajo en escuela, vínculo familia-escuela, ventajas y desventajas de la herramienta visita domiciliaria y herramientas necesarias para el psicólogo que trabaja en dupla escolar.

La metodología utilizada mantiene la sensibilidad y apertura a los contenidos específicos de cada caso, sin embargo, como limitante debe considerarse que las comparaciones entre grupos están basadas teóricamente en un proceso o problemática específica, por lo que el análisis se restringe a ello (Flick, 2012). Los datos obtenidos a partir de las entrevistas y discusión grupal fueron triangulados con antecedentes teóricos, este proceso brindó mayor solidez metodológica a los resultados obtenidos (Martínez, 2006).

Dentro de las consideraciones éticas, a todos los participantes se les entregó un consentimiento informado en el cual se detallaron los objetivos, procedimiento, costos del estudio, condición voluntaria de su participación y confidencialidad sobre las opiniones entregadas. Por último, a la Corporación de Educación Municipal y a los participantes del estudio se les envió un informe ejecutivo con los principales resultados obtenidos a modo de retribución.

\section{RESULTADOS}

A partir del análisis emergen seis categorías relevantes sobre el trabajo en el ámbito psicosocial que realizan los profesionales entrevistados.

\subsection{PRAXIS DE LA DUPLA Y EQUIPO PSICOSOCIAL EN TERRENO}

Las actividades realizadas por el EP según los entrevistados son variadas y dependen de la contingencia de la escuela, la cual se describe como un ambiente muy dinámico. No hay una delimitación clara sobre qué actividades corresponden a la dupla y cuáles no, por lo que las actividades se asignan según lo que interpreta la corporación de educación municipal, directivos y las propias duplas (ver Tabla 2). Por lo tanto, el producto de intervenciones psicosociales varía en cada escuela según el grado de influencia que tiene: la necesidad del contexto, lineamientos de la escuela, directivos, profesionales y lineamientos de la municipalidad.

La baja de delimitación del cargo del EP según los entrevistados es una ventaja para la escuela, ya que así pueden contar con los profesionales como un recurso disponible 
para casi cualquier actividad. Por otro lado, los mismos entrevistados declaran haberse creado sus propias pautas, metas, objetivos y materiales para el trabajo psicosocial, lo cual resta tiempo (recurso escaso) y diversifica la intervención psicosocial escolar. Funcionan entonces interviniendo en múltiples áreas, pero sin continuidad en ninguna. Los profesionales ocupan la metáfora de estar constantemente "apagando incendios" (ver Tabla 2), lo cual se traduce en que están constantemente resolviendo crisis, debido a la alta complejidad del contexto y casos críticos, lo cual les resta tiempo para desarrollar actividades a largo plazo.

Desde el punto de vista de los entrevistados, esta percepción difusa de las actividades que les corresponden y poca continuidad genera poca visibilidad del impacto del trabajo psicosocial en la comunidad escolar (ver Tabla 2). Esto deriva en que se le resta prioridad ya que se prefiere atender otras urgencias en la escuela. Los entrevistados observan que no se le otorga el espacio ni tiempo necesario al trabajo psicosocial, por ejemplo, en el Consejo de Profesores no se reserva un tiempo para asuntos psicosociales, ya que los temas de interés son orientados al rendimiento académico y financiamiento. Esto dificulta la comunicación entre EP y profesores, ya que no existen otros tiempos para poder coordinarse.

En concreto dentro de las actividades que declaran realizar los equipos psicosociales, las más frecuentes son; vinculación escuela-familia, vinculación con redes, casuística, desarrollo de talleres dirigido a niños o apoderados, formación de profesores en el área psicosocial, apoyo al inspector en el recreo, intervención en crisis, entrevistas con apoderados, cuidar salas, incluso organización de actividades recreativas, etc.

Según la percepción de los entrevistados, la diversidad de áreas en la que el equipo psicosocial interviene trae por consecuencia que demandas diarias de la escuela sobrepase el tiempo y capacidad que tiene el profesional para abordarlas. Si lo sumamos a la realidad de que muchos estudiantes por los programas de integración presentan necesidades especiales y además contexto de alta vulnerabilidad, trae por consecuencia que el profesional sienta una sobrecarga laboral y que muchas veces tenga que dedicar horas extra (ver Tabla 2). 
Tabla 2. Citas sobre la praxis del Equipo Psicosocial en escuelas

\begin{tabular}{|c|c|c|}
\hline Categoría & Subcategoría & Citas \\
\hline \multirow{7}{*}{$\begin{array}{l}\text { Praxis del } \\
\text { EP o DP }\end{array}$} & $\begin{array}{l}\text { Actividades } \\
\text { asignadas } \\
\text { varían }\end{array}$ & $\begin{array}{l}\text { “(...) muchas veces estos equipos son delineados desde las } \\
\text { corporaciones, otras veces los directores (...)” (Entrevista } 3 \text {, } \\
\text { párrafo 88). } \\
\text { “(..) No hay un perfil, no hay, o sea, más que un perfil no hay como } \\
\text { una ruta de trabajo general para todos, "las duplas trabajan así” } \\
\text { ¿Cachay? No, cada escuela pone o ve cuales son las necesidades } \\
\text { del Colegio (...)” (Entrevista 6, párrafo 47). }\end{array}$ \\
\hline & \multirow{2}{*}{$\begin{array}{l}\text { Improvisación } \\
\text { - "apagando } \\
\text { incendios" }\end{array}$} & $\begin{array}{l}\text { “(..) le pregunté al orientador "oye, ¿qué hacía mi colega } \\
\text { anteriormente? ¿Qué puedo mirar?” "chuta no sé, yo veía que } \\
\text { atendía niños, entrevistaba, iba como al aula...” y me inventé. Me } \\
\text { inventé un panorama” (Entrevista grupal, párrafo 76). }\end{array}$ \\
\hline & & $\begin{array}{l}\text { "O apagando incendios o focalizada en el caso. El caso siempre va } \\
\text { a estar, pero la escuela no mira esta cosa, esta problemática social } \\
\text { de manera más a largo plazo (...)" (Entrevista grupal, párrafo 43). }\end{array}$ \\
\hline & \multirow{3}{*}{$\begin{array}{l}\text { Percepción de } \\
\text { bajo impacto } \\
\text { de las } \\
\text { intervenciones } \\
\text { psicosociales }\end{array}$} & $\begin{array}{l}\text { “(...) no están los tiempos, entonces muchas cosas tú las haces de } \\
\text { pasillo (...) las intervenciones tienen que ser rápidas, pero muchas } \\
\text { veces tampoco son tan efectivas, porque quizás, a uno le gustaría } \\
\text { abordarlo de otra manera, pero en realidad la escuela no lo } \\
\text { acompaña." (Entrevista grupal, párrafo 26). }\end{array}$ \\
\hline & & $\begin{array}{l}\text { "Se siente como que no hay impacto, claro que no hay impacto, } \\
\text { pero (...) tiene que ver con eso de que no es claro (...)." (Entrevista } \\
6 \text {, párrafo 58). }\end{array}$ \\
\hline & & $\begin{array}{l}\text { "No nos empodera, al final vamos a ser canapés" (...) para que } \\
\text { digamos "si, cumplimos la meta", cumplimos con el PME, } \\
\text { cumplimos. Pero que efectivamente uno [no] hizo la pega que } \\
\text { debiese haber hecho con el impacto socioeducativo que uno } \\
\text { esperaría (...)" (Entrevista grupal, párrafo 56). }\end{array}$ \\
\hline & $\begin{array}{l}\text { Horas extra y } \\
\text { sobrecarga } \\
\text { laboral }\end{array}$ & $\begin{array}{l}\text { "Pero de repente creo que también es una sobrecarga de trabajo } \\
\text { (...) porque todo [es] convivencia [escolar]" (Entrevista grupal, } \\
\text { párrafo } 77 \text { ). }\end{array}$ \\
\hline
\end{tabular}

* Modismo chileno, metáfora utilizada para referirse a una persona que se encuentra en muchos lugares, pero realmente no hace grandes aportes al medio. Similar al aperitivo "canapé" los cuales se distribuyen en grandes cantidades, pero al ser pequeños bocados no sacian al invitado.

Fuente: elaboración propia. EP: Equipo Psicosocial, DP: Dupla Psicosocial.

\subsection{ROL DEL EQUIPO PSICOSOCIAL Y PSICÓLOGO EN CONTEXTO ESCOLAR}

Los hallazgos permiten sostener que hay poca claridad sobre qué es y para qué sirve la Dupla psicosocial. Según las entrevistas la descripción de cargo se entrega desde la municipalidad estableciendo que el foco de la dupla es convivencia escolar, retención escolar y trabajar promoción y prevención. Pero en la práctica se observa poca delimitación del concepto 
de convivencia escolar, bajo entendimiento por el personal de la escuela sobre el rol y actividades que corresponden a la dupla y funcionamiento reactivo ante la realidad que se contrapone al enfoque preventivo.

Al preguntarles directamente su rol a las duplas estas se identifican con la labor de asegurar el bienestar biopsicosocial del niño/a, generar en la escuela un lugar protector, vincular familia-escuela y generar vínculos con redes tanto internas como externas. Esto se desprende de lo que ellos interpretan como convivencia escolar (ver Tabla 3).

El propósito de conformar un equipo psicosocial (usualmente conformada por psicólogo/a y trabajador/a social) es generar intervenciones en conjunto para lograr mejor calidad, ya que se aborda el caso desde distintas aristas según lo que aporta la experticia de cada profesión. Esto amplía la mirada del profesional y genera intervenciones de mejor calidad requiriendo habilidades de trabajo en equipo (ver Tabla 3).

A partir de la confusión del concepto de dupla, surgen expectativas y estereotipos sobre el trabajo de estos dentro de la comunidad escolar que no suelen ser realistas (ver Tabla 3 ). Por lo que la asignación de tareas y la posterior evaluación de la dupla es compleja, dado que no hay indicadores que reflejen el trabajo del equipo psicosocial salvo una encuesta de convivencia escolar. Sobre el rol del psicólogo/a escolar suele confundirse con la labor clínica, esta expectativa errónea puede provenir tanto de profesores, directivos, instituciones como incluso psicólogos/as que se están recién insertando el ámbito escolar. Respecto a la labor del trabajador/a social suele simplificarse a otorgar becas, beneficios materiales para los niños/as y a visitas domiciliarias. Estas ideas son estereotipadas y limitan el alcance de estas profesiones, sobre el aporte que pueden entregar al trabajo socioeducativo.

Un elemento que destaca a partir de las entrevistas sobre el rol de la DP y EP es que los participantes consideran fundamental abordar las problemáticas en las escuelas desde una mirada comunitaria (ver Tabla 3). La intervención se aborda desde esta perspectiva ya que permite ver al estudiante no solo como individuo, sino como parte de múltiples ambientes y redes que intervienen y confluyen. Por lo que el lineamiento de la escuela se orienta a trabajar desde la conexión y comunicación entre estas múltiples redes de soporte existentes en los sistemas en los que interactúa el niño/a. Es entonces cuando la dupla participa como elemento articulador y trabaja facilitando el trabajo en red. 
Tabla 3. Citas sobre el rol del equipo psicosocial en contexto educativo

\begin{tabular}{|c|c|c|}
\hline Categoría & Subcategoría & Citas \\
\hline \multirow{5}{*}{$\begin{array}{l}\text { Rol de la } \\
\text { DP/EP y } \\
\text { psicólogo/a } \\
\text { en contexto } \\
\text { educativo }\end{array}$} & $\begin{array}{l}\text { Definición difusa } \\
\text { de convivencia } \\
\text { escolar }\end{array}$ & $\begin{array}{l}\text { "Entonces si tú me preguntas cuál es el rol, oficialmente puede } \\
\text { estar más acotado, pero en la práctica es como todo (...)" } \\
\text { (Entrevista 2, párrafo 61). }\end{array}$ \\
\hline & $\begin{array}{l}\text { DP se identifican } \\
\text { con asegurar el } \\
\text { bienestar } \\
\text { biopsicosocial } \\
\text { del niño/a }\end{array}$ & $\begin{array}{l}\text { "evitar la deserción escolar en los chicos. Y también ir avanzando } \\
\text { como hacia un clima de convivencia (...) Considerando que este } \\
\text { es un espacio donde muchos de los chicos son vulnerados en sus } \\
\text { derechos y no tienen muchos espacios más que este, donde estén } \\
\text { protegidos y como contenidos y donde puedan ir desarrollándose } \\
\text { también como personas" (Entrevista 5, párrafo 66). }\end{array}$ \\
\hline & $\begin{array}{l}\text { EP como } \\
\text { estrategia } \\
\text { interdisciplinaria }\end{array}$ & $\begin{array}{l}\text { “yo siento que la lógica es generar diferentes miradas, (...) } \\
\text { siendo las dos de las ciencias sociales manejamos cosas en común } \\
\text { y podemos generar intervenciones en común (...).” (Entrevista } 2 \text {, } \\
\text { párrafo 61). }\end{array}$ \\
\hline & $\begin{array}{l}\text { Expectativas y } \\
\text { estereotipos } \\
\text { sobre el trabajo } \\
\text { psicosocial por } \\
\text { la comunidad } \\
\text { escolar }\end{array}$ & $\begin{array}{l}\text { "(...) no se entiende el rol de nosotros ¿me entiendes? porque al } \\
\text { final se entiende ah una Asistente Social, ya para que le diga lo de } \\
\text { la beca al niño, para que le consiga los lentes ¿cachay? Ah la } \\
\text { Psicóloga, ah vea a este niñito que debe tener problemas } \\
\text { emocionales ¿Entendí? entonces como que está separado, no } \\
\text { entienden.” (Entrevista 6, párrafo 45). } \\
\text { ” No se sabe mucho qué es lo que hace la dupla o los equipos } \\
\text { vinculados con el mundo de las ciencias sociales en la escuela; sí, } \\
\text { los profesores tienen un imaginario ahí bastante florido en } \\
\text { relación a eso y muchas veces muy prejuicioso. Pero también los } \\
\text { profesionales que se instalan en las escuelas tampoco saben muy } \\
\text { bien que es lo que tienen que hacer” (Entrevista 3, párrafo 24). }\end{array}$ \\
\hline & $\begin{array}{l}\text { Mirada } \\
\text { comunitaria }\end{array}$ & $\begin{array}{l}\text { "Pasar de lo individual a lo más comunitario que es todo un } \\
\text { proceso, porque tiene que ver con la vinculación con las diferentes } \\
\text { áreas, con las diferentes redes, para mirar un problema" } \\
\text { (Entrevista grupal, párrafo 26). } \\
\text { "(...) convocamos a las distintas redes que giran en torno a la } \\
\text { escuela para abordar temas que sirvan de herramienta a los } \\
\text { apoderados en su relación con los chiquillos (...)" (Entrevista 3, } \\
\text { párrafo 46). }\end{array}$ \\
\hline
\end{tabular}

Fuente: elaboración propia. EP: Equipo Psicosocial, DP: Dupla Psicosocial. 


\subsection{COMPETENCIAS}

Se extrae del análisis habilidades destacadas necesarias para el trabajo en las escuelas del estudio (ver Tabla 4). En primer lugar, se menciona el tener experiencia, apertura o haber pertenecido en su origen a un barrio vulnerable (a lo que algunos llamaron "tener calle"). Esto apunta a que hay conocimientos prácticos sobre el contexto que en la teoría no es posible enseñar. Al tener experiencias en este tipo de barrios el profesional adquiere herramientas para desenvolverse y sentirse seguro al interactuar con el espacio.

Otra característica relevante es la capacidad de empatía con familias y niños/as, según los entrevistados, es indispensable para poder ser sensible a la realidad cultural del niño/a, esto permite ser comprensivos con la situación de la familia, vincularse con ellos y poder detectar los recursos que la familia tiene para ser potenciados (ver Tabla 4). Esta habilidad se relaciona con otra habilidad mencionada el cual los entrevistados llaman "aperrar", la cual se define como la disposición de trabajar intensamente por los niños/as, y se podría traducir en la vocación que tiene el profesional de dedicar su trabajo al bienestar infantil en contextos desafiantes (ver Tabla 4).

Por último, los entrevistados destacan como parte importante del perfil del profesional para el trabajo que ejercen el trabajo en equipo y tolerancia a la frustración. Esto se debe principalmente a que en el espacio educativo convergen distintas disciplinas, y por lo tanto la capacidad de dialogo y trabajo en equipo se vuelve indispensable para realizar un trabajo colaborativo de calidad (ver Tabla 4).

Tabla 4. Citas sobre competencias para el trabajo en Dupla Psicosocial en escuelas municipales

\begin{tabular}{|c|c|c|}
\hline Categoría & Subcategoría & Citas \\
\hline \multirow{5}{*}{$\begin{array}{l}\text { Competencias } \\
\text { del EP y } \\
\text { psicólogo/a } \\
\text { educacional }\end{array}$} & "Tener Calle" & $\begin{array}{l}\text { "Debería tener así experiencia en barrios, bueno igual es algo que } \\
\text { se puede ir adquiriendo, pero a mí la experiencia me dice que tiene } \\
\text { que ver igual con gente que ya ha estado en barrios (...) que no te } \\
\text { las va a dar el trabajar si no que tení que haberlas vivido no más" } \\
\text { (Entrevista 1, párrafo 178). }\end{array}$ \\
\hline & & $\begin{array}{l}\text { “(...) También es una de las poblaciones más conflictivas de la } \\
\text { comuna, pero yo soy de acá, no de aquí de la Castrina*, pero sí } \\
\text { de San Joaquín” (Entrevista 4, párrafo 146). }\end{array}$ \\
\hline & $\begin{array}{l}\text { Empatía hacia } \\
\text { familias y } \\
\text { niños/as }\end{array}$ & $\begin{array}{l}\text { “(...) pa' mí siempre tratar de empatizar un poco con las personas } \\
\text { que voy a realizar las entrevistas, pero también siempre teniendo } \\
\text { en cuenta a los niños y observando las dinámicas que ahí se dan } \\
\text { en pro del beneficio de ellos.” (Entrevista 3, párrafo 244). }\end{array}$ \\
\hline & $\begin{array}{l}\text { Disposición a } \\
\text { "aperrar" }\end{array}$ & $\begin{array}{l}\text { "que sea como capaz de como de jugarse un poco el pellejo por } \\
\text { los niños. No tan literal, pero si estar como dispuesta a usar un } \\
\text { poco más de tiempo de lo que debería (...)” (Entrevista } 5 \text {, párrafo } \\
55 \text { ). }\end{array}$ \\
\hline & $\begin{array}{l}\text { Trabajo en } \\
\text { equipo }\end{array}$ & $\begin{array}{l}\text { "Primero tiene que ser capaz de trabajar en equipo, esa la } \\
\text { habilidad laboral. Y alta tolerancia a la frustración, porque en } \\
\text { realidad se abordan problemáticas que son sociales" (Entrevista } \\
6, \text { párrafo 49). }\end{array}$ \\
\hline
\end{tabular}

*Barrio dentro de la comuna de San Joaquín, Región Metropolitana, Santiago de Chile.

Fuente: elaboración propia. EP: Equipo Psicosocial. 


\subsection{DESAFÍOS RESPECTO AL TRABAJO DEL EQUIPO PSICOSOCIAL}

Como ya fue caracterizado, dentro de la comuna de estudio existen poblaciones con altos niveles de violencia, vulnerabilidad y marginación. Esto afecta de forma multicausal a los niños y niñas y dirige las líneas de trabajo de la escuela. Debido a esto, los equipos reciben diariamente niños desestabilizados quienes presentan altos niveles de hiperactividad, violencia, descontrol de impulsos, problemas neurológicos, entre otros. Esto presenta un desafío importante ya que expone al profesional a diario a situaciones de agresividad (ver Tabla 5).

Al trabajar con las familias de estos niños y niñas, el profesional declara encontrar en general alto grado de escepticismo y baja colaboración inicial. Según los entrevistados se observa desesperanza aprendida y desconfianza sobre el funcionamiento del servicio público (Tabla 5). Los entrevistados especulan que esto puede deberse a experiencias frustrantes en servicios públicos y/o sobre intervención estatal. Esto hace desafiante el vínculo y generar un compromiso con la institución educativa.

Como obstáculos en la visita, los entrevistados consideran que no se toman precauciones para la seguridad personal del visitador. Dado que los barrios que se visitan son de alta complejidad, los Equipos Psicosociales se preocupan de ir acompañados (entre ellos o con redes) e informan constantemente su ubicación, sin embargo, persiste esta sensación de inseguridad (Tabla 5). Generalmente los hogares se encuentran cerca de la escuela, por lo que los visitadores se trasladan caminando, pero existen domicilios situados en poblaciones de mayor complejidad a las cuales se accede solo en automóvil o colectivos financiados por los mismos profesionales para resguardar su propia seguridad.

Por último, como los entrevistados consideran sumamente relevante poder balancear la necesidad de la institución educativa y los intereses e indicadores externos a la escuela. La Dupla funciona como un elemento articulador entre distintos niveles, por lo que debe ser capaz de mediar entre los intereses de cada parte, distribuir los tiempos y priorizar intervenciones. Un ejemplo observado es que durante el programa "Aquí Presente" ${ }^{3}$ hubo modificaciones importantes, donde profesionales prefirieron retirarse de las escuelas. El problema surgía cuando la dupla no estaba en sintonía con las necesidades de la institución, de ello dependía cuan valorada e integrada se considera dentro de la comunidad escolar (ver Tabla 5).

\footnotetext{
"Aquí presentes" corresponde a un programa del Gobierno Regional Metropolitano, ejecutado por el Ministerio de Educación, que tiene por objetivo reducir la deserción escolar mediante la implementación de duplas psicosociales en distintas escuelas de la Región Metropolitana. Se busca fomentar la buena convivencia y el buen trato, con el fin de identificar las señales de alerta de deserción escolar e incrementar la asistencia de los y las estudiantes identificados generando redes de apoyo en la comunidad e intervención psicosocial (http://aquipresente.mineduc.cl). (debe quedar la nota en la misma página).
} 
Tabla 5. Citas sobre desafíos en la praxis del equipo psicosocial y psicólogo/a en escuelas municipales

\begin{tabular}{|c|c|c|}
\hline Categoría & Subcategoría & Citas \\
\hline \multirow{6}{*}{$\begin{array}{l}\text { Desafíos del } \\
\text { EP y } \\
\text { psicólogo/a } \\
\text { educacional }\end{array}$} & $\begin{array}{l}\text { Exposición del } \\
\text { profesional a } \\
\text { altos niveles de } \\
\text { violencia }\end{array}$ & $\begin{array}{l}\text { "a mí me ha tocado atender niños que sacan una fuerza (...) y } \\
\text { olvídate tu no podí tomar a ese niño, te acercai y te pega y te pega } \\
\text { patá", te escupe, te muerde, y además tiene mucha fuerza. Es un } \\
\text { nivel de descontrol súper grave" (Entrevista 6, párrafo 215). }\end{array}$ \\
\hline & $\begin{array}{l}\text { Familias con } \\
\text { alto grado de } \\
\text { escepticismo y } \\
\text { baja } \\
\text { colaboración } \\
\text { inicial }\end{array}$ & $\begin{array}{l}\text { “(..) que hay familias reticentes a no entregar información, de } \\
\text { repente que el desafío es cómo "hincar el diente”* para que te } \\
\text { digan cosas” (Entrevista 4, párrafo 142). }\end{array}$ \\
\hline & $\begin{array}{l}\text { Sensación de } \\
\text { riesgo y falta de } \\
\text { financiamiento } \\
\text { en traslados }\end{array}$ & $\begin{array}{l}\text { “(...) la mayoría de las familias viven acá cerquita, por acá por } \\
\text { la misma calle acá, mas pábajo, en la Legua de Emergencia, } \\
\text { ahí vive, yo diría que el } 80 \% \text { de los niños que vienen acá.” } \\
\text { (Entrevista 1, párrafo 101). }\end{array}$ \\
\hline & & $\begin{array}{l}\text { "nosotras nos movemos en territorios ultra complejos, muy } \\
\text { complejos. No tenemos tarjeta BIP**, no tenemos plata para } \\
\text { colectivo, y andamos a pata y muchas veces solas." (Entrevista } \\
\text { 2, párrafo 215). }\end{array}$ \\
\hline & $\begin{array}{l}\text { Sintonía con las } \\
\text { necesidades de } \\
\text { la institución }\end{array}$ & $\begin{array}{l}\text { "Ese ha sido el desafío honestamente siendo bien sincera, al } \\
\text { programa, yo creo, poco le importa lo que está pasando aqui en } \\
\text { la escuela. Al programa le interesan las cosas que le interesan al } \\
\text { programa, que las cosas se hagan, si hay reuniones tenemos que } \\
\text { ir, entonces no se considera mucho la necesidad que tiene la } \\
\text { escuela y tampoco se conoce cien por ciento el trabajo que uno } \\
\text { hace acá en la escuela" (Entrevista } 1 \text {, párrafo 59). }\end{array}$ \\
\hline & & $\begin{array}{l}\text { "A nosotros se nos dijo desde un comienzo que la dupla se iba a } \\
\text { adecuar a las necesidades de la escuela (...) Y, bueno, cuando } \\
\text { llegó la dupla empezó a instalarse otra lógica, así que ellos } \\
\text { tenían que funcionar en relación con lo que el Ministerio estaba } \\
\text { señalando. Entonces obviamente tuvimos nuestros encontrones } \\
\text { en relación a eso y la dupla terminó yéndose de acá." (Entrevista } \\
\text { 3, párrafo 36). }\end{array}$ \\
\hline
\end{tabular}

* Modismo chileno, metáfora para referir la necesidad de presionar a otro para que realice una acción.

** Tarjeta requerida para utilizar el transporte público en Santiago de Chile.

Fuente: elaboración propia. EP: Equipo Psicosocial. 


\subsection{DESARROLLOY VALORACIÓN DE LA VISITA DOMICILIARIA ESCOLAR}

La visita domiciliaria es una herramienta valorada positivamente por los participantes del estudio. Se utiliza para ampliar la mirada en el trabajo de casuística, ya que permite observar elementos importantes del ambiente hogar (físico y relacional), por lo que enriquece la información y complementa la observación en escuela (Tabla 6).

Por lo general se realiza en el interior de la vivienda. Idealmente se busca hablar con el apoderado del niño/a, en caso de que no se encuentre, se utiliza la instancia para hablar con cualquier persona del entorno que pueda aportar con información relevante al caso como: condiciones de vivienda, relaciones familiares, rutinas de la casa, rubro en el que se desempeña el jefe de hogar o familia, motivos de inasistencias del alumno, entre otros (Tabla 6). Aproximadamente estas visitas se extienden en promedio $45 \mathrm{~min}$.

Los principales motivos de la visita según las entrevistas son deserción escolar y sospecha de vulneración de derechos del niño/a. Esta herramienta se utiliza principalmente para elaborar un mejor diagnóstico de la situación familiar, establecer un vínculo familiaescuela, intervención psicoeducativa, rescate y protección de los derechos del niño/a. La dupla pretende dejar el estigma de la escuela como "punitiva" para generar un vínculo más horizontal (Tabla 6).

Tabla 6. Citas sobre el uso de la Visita Domiciliaria por el Equipo Psicosocial

\begin{tabular}{|c|c|c|}
\hline Categoría & Subcategoría & Citas \\
\hline \multirow{4}{*}{$\begin{array}{l}\text { Visita } \\
\text { Domiciliaria }\end{array}$} & $\begin{array}{l}\text { Ampliar la mirada } \\
\text { sobre el caso }\end{array}$ & $\begin{array}{l}\text { "Porque eso, iclaro!, te permitiría tener una panorámica } \\
\text { mucha más amplia de que es lo que ocurre con ellos, mucha } \\
\text { más frecuencia porque creo es una herramienta muy } \\
\text { importante" (Entrevista 3, párrafo 254). }\end{array}$ \\
\hline & $\begin{array}{l}\text { Lugar donde se } \\
\text { desarrolla la VDE }\end{array}$ & $\begin{array}{l}\text { "Yo siempre intento que me hagan pasar, cachay. Hasta, así } \\
\text { como pidiendo un vaso de agua, porque igual al final en la } \\
\text { visita domiciliaria tienes que ver la casa por dentro cachay, } \\
\text { como que la idea también es ver las condiciones de vida de } \\
\text { la familia" (Entrevista 5, párrafo 200). }\end{array}$ \\
\hline & Motivos de la VDE & $\begin{array}{l}\text { "El típico es asistencia (...) Entonces el objetivo es } \\
\text { finalmente como conversar con la familia, explicarle como, } \\
\text { a lo que se expone finalmente si el chico deserta de la } \\
\text { educación" (Entrevista 5, párrafo 211). }\end{array}$ \\
\hline & $\begin{array}{l}\text { Generar vínculo } \\
\text { horizontal con la } \\
\text { familia }\end{array}$ & $\begin{array}{l}\text { “(...) nos dimos cuenta que cuando se contactaba a la familia } \\
\text { desde la escuela era un enfoque más punitivo, "bueno señora } \\
\text { Ud. es floja”, a muchas familias se les amenazaba con } \\
\text { tirarles a los carabineros para que mandaran a sus hijos y } \\
\text { nosotros eso lo descartamos porque encontramos que esa no } \\
\text { es la forma (...)." (Entrevista } 1 \text {, párrafo } 40 \text { ). }\end{array}$ \\
\hline
\end{tabular}

Fuente: elaboración propia. VDE: Visita Domiciliaria Escolar. 


\subsection{BARRERAS INSTITUCIONALES DETECTADAS}

En los discursos recogidos, se genera confusión respecto a las intervenciones psicosociales ya que los entrevistados declaran una falta de guías prácticas para la acción (Tabla 7). Desde la Corporación de Educación se formulan algunos lineamientos para la implementación, pero estos son optativos para los establecimientos, por lo que no asegura la homogeneidad de las intervenciones. Las actividades varían según comuna, escuela y profesional. Sobre las estrategias a implementar dado que no hay lineamientos oficiales, los profesionales improvisan y experimentan para decidir cuáles son las más apropiadas a cada situación.

Un factor que considerar es la permanencia de algunas duplas en escuelas, ya que estas dependen del financiamiento de programas externos los cuales no son estables en el tiempo. Esto significa que las intervenciones psicosociales no tienen asegurada su continuidad, lo cual pueden generar resultados iatrogénicos. Las duplas explican que en el caso de los programas con fondos públicos dependen directamente de la situación política que se vive. Independientemente a los resultados obtenidos por el programa, la renovación de fondos depende de la figura política que los representa (Tabla 7).

Otras barreras institucionales comentadas, es que para solicitar autorizaciones o recursos se pierde mucho tiempo en la burocracia de los sistemas públicos, por lo que finalmente los equipos optan por ocupar sus propios medios o solo lo que esté disponible en la escuela. Los establecimientos facilitan espacios, pero no cuentan con suficientes recursos materiales (Tabla 7).

Tabla 7. Citas sobre barreras institucionales en el trabajo de Equipos Psicosociales en escuelas municipales

\begin{tabular}{|c|c|c|}
\hline Categoría & Subcategoría & Citas \\
\hline \multirow{3}{*}{$\begin{array}{l}\text { Barreras } \\
\text { institucionales }\end{array}$} & $\begin{array}{l}\text { Falta de guías } \\
\text { prácticas }\end{array}$ & $\begin{array}{l}\text { "(...) No hay un perfil, no hay o sea más que un perfil, no hay } \\
\text { como una ruta de trabajo general para todos, "las duplas } \\
\text { trabajan asi”", ¿cachay? No, cada escuela pone su... o ve } \\
\text { cuales son las necesidades del Colegio y ve de que le sirve } \\
\text { (...)" (Entrevista 6, párrafo 47). }\end{array}$ \\
\hline & $\begin{array}{l}\text { Rotación de } \\
\text { programas por } \\
\text { contexto } \\
\text { político }\end{array}$ & $\begin{array}{l}\text { "Si aquí hay política, como en todas las cosas, hay un eje } \\
\text { político muy potente detrás. Piensa que por ejemplo el loco } \\
\text { que estaba casado con este programa es O., (...) tiene } \\
\text { demasiados enemigos políticamente, entonces el hecho que él } \\
\text { se haya casado con este programa fue un arma de doble filo } \\
\text { desde el principio se pierde el foco (...)" (Entrevista } 1 \text {, } \\
\text { párrafo 360). }\end{array}$ \\
\hline & $\begin{array}{l}\text { Burocracia en } \\
\text { sistemas } \\
\text { públicos para } \\
\text { obtener } \\
\text { recursos y } \\
\text { autorizaciones }\end{array}$ & $\begin{array}{l}\text { “(..) hay espacio y si requerimos alguna cosa, material, } \\
\text { también, cuando se puede se hace (...) Es que eso no depende } \\
\text { solo de la dirección, depende también de la corporación } \\
\text { muchas veces y de la burocracia que hay ahî" (Entrevista } 3 \text {, } \\
\text { párrafo } 102,104) \text {. }\end{array}$ \\
\hline
\end{tabular}

Fuente: elaboración propia. 


\section{DISCUSIÓN Y CONCLUSIONES}

Este estudio logra ser un primer acercamiento a la realidad de los equipos psicosociales en las escuelas donde se observa que existe gran dificultad en comprender y traspasar los conceptos fundamentales descritos en las reformas educacionales chilenas en la ejecución en las escuelas.

En concordancia con la bibliografía, se observa que la intervención psicosocial es una necesidad reconocida por el sistema educativo, pero presenta bajo apoyo institucional (Gatica \& Alfaro, 2015). En esta investigación se encontraron distintas barreras que dificultan el trabajo del equipo psicosocial en escuelas, tales como: falta de comprensión sobre el rol de los quipos psicosociales; escasa delimitación del cargo y perfil de trabajo; heterogeneidad en las intervenciones; falta de concordancia entre necesidades de la escuela y metas políticas; baja percepción del impacto de las intervenciones psicosociales; expectativas erradas y estereotipos que limitan el trabajo psicosocial; entre otras.

En el presente estudio, se observa que la falta de delimitación del perfil profesional conlleva a que el recurso de equipo psicosocial sea utilizado para cubrir otras necesidades de la escuela. Esto se traduce en que las actividades responden al contexto, pero no necesariamente a los objetivos propuestos por las reformas políticas (cuidar aulas, apoyar a las funciones del inspector en el recreo, organizar actividades recreativas, etc.) Como ya fue señalado por Gatica y Alfaro (2015), esta realidad pone en tensión las metas y objetivos en el área psicosocial, siendo diferentes las demandas de la escuela con lo propuesto por el Estado. La falta de concordancia entre nivel político y ejecución afecta la calidad de las intervenciones para bienestar psicosocial (Gatica y Alfaro, 2015).

Por otro lado, se debe comprender que la educación municipal se enfrenta a un contexto demandante, donde las necesidades sobrepasan con creces los recursos. Para enfrentar esta realidad las escuelas funcionan de forma reactiva, es decir, priorizan los casos más graves dejando de lado las intervenciones preventivas. Se genera entonces, un círculo no virtuoso, ya que la literatura existente afirma que las intervenciones sostenidas en el tiempo y las de carácter preventivo son más efectivas para abordar problemáticas multicausales en contextos de vulnerabilidad (Siverio, 2011; MINSAL, 2013; Saracostti, 2013).

A nivel político se espera que el equipo psicosocial sea un "agente de cambio" en la escuela, proponiendo nuevas estrategias, capacitando al equipo docente, instalado una visión biopsicosocial, entre otras actividades. Al mismo tiempo, las duplas deben responder a las expectativas y necesidades de la comunidad educativa que los acoge. Por lo tanto, es la dupla psicosocial la que recibe la difícil tarea de abordar el cambio de paradigma (desde reactivo a preventivo). Las duplas deben cuestionar la dinámica ya instalada, siendo minoría y nuevos actores en el mundo educativo.

Uno de los elementos destacables de los resultados, es el enfoque comunitario en el trabajo de los entrevistados. Este concepto potencia el rol de la dupla psicosocial como elemento articulador para el trabajo en red (sistema de salud, red de protección de la infancia, centros comunitarios, privados, entre otros). En Arévalo y Santana (2013), se define el concepto de la educación como un elemento multicausal, por lo que cada vez se hace más necesario el abordaje desde un enfoque comunitario. Lo cual hace relevante que futuras investigaciones puedan profundizar en el trabajo intersectorial y como este podría beneficiar la intervención psicosocial que se realiza actualmente en escuelas chilenas. 
Por último, la visita domiciliaria escolares una herramienta valorada por los equipos para ampliar la mirada en la casuística y entablar un vínculo horizontal con la familia. Esta herramienta es utilizada para abordar casos de protección de la infancia, convirtiéndola en un elemento con potencial para beneficiar la calidad de la educación municipal (Razeto, 2016). Al comparar las visitas domiciliarias en Chile con los programas internacionales descritas por Cowan et al. (2002) y Meyer et al. (2011), se observa que nuestra realidad realiza con menor frecuencia en las visitas y el énfasis está puesto en diagnóstico y rescate.

A partir del estudio se concluye que no se comprende el rol del equipo psicosocial en las escuelas generando diversas barreras al momento de realizar intervenciones psicosociales. La escuela presenta un funcionamiento reactivo a sus necesidades internas, por lo que trabajan con estrategias propias basadas en la experiencia, siendo resistentes a los nuevos abordajes definidos por entidades externas a la escuela. Además, la optimización de los recursos y el tiempo profesional limita la innovación y pilotaje de nuevas intervenciones.

Sobre los alcances y limitaciones, este estudio logra ser una de las primeras aproximaciones al fenómeno de dupla o equipo psicosocial en contexto educativo de Chile. Además de ser una de las primeras investigaciones en reflexionar sobre las potencialidades de la visita domiciliaria escolar en el país. Por otro lado, esta investigación se realiza bajo una la metodología cualitativa la cual otorga grandes ventajas sobre la profundización de la realidad, aunque sus resultados deben ser entendidos bajo las limitaciones cualitativas y no se puede generalizar a una realidad país. Respecto a las técnicas de producción de datos, aunque estas cumplen satisfactoriamente con el diseño, para investigaciones futuras se sugiere agregar más técnicas (observación en terreno, grupo focal, entre otras). También se sugiere incluir más actores de la comunidad escolar ales como profesores, familias, estudiantes, directivos y entidades municipales. Esto lograría enriquecerlas perspectivas de la realidad psicosocial escolar. Finalmente debe considerarse la alta rotación de profesionales en escuelas municipales y la reciente incorporación de equipos psicosociales, ya que gran parte de los reportes corresponden al trabajo de menos de un año académico pudiendo sesgar las opiniones de los entrevistados.

Para futuras investigaciones se evidencia la necesidad en Chile de generar orientaciones técnicas e investigaciones para la intervención psicosocial en escuelas con enfoque intersectorial, adaptadas al contexto dinámico y que consideren la circularidad de los procesos educativos.

\section{REFERENCIAS BIBLIOGRÁFICAS}

Allen, S. \& Tracy, E. (2004). Revitalizing the role of home visiting by school social workers. Chillaren and Schools, 26(4), 197-208.

Aracena, M., Leiva, L., Undurraga, C., Krause, M., Pérez, C., Cuadra, V., Campos, M. \& Bedregal, P. (2011). Evaluación de la efectividad de programas de visitas domiciliarias para madres adolescentes y sus hijos. Revista Médica de Chile, 139, 60-65.

Arévalo, M. \& Santana, A. (2013). Percepción de los resultados de la intervención social implementada por duplas psicosociales en escuelas municipales. Implicancia de la vinculación familia-escuela. En: Saracostti, M. (Ed.). Familia-Escuela Comunidad III: una alianza necesaria para un modelo de intervención biopsicosocial positivo. (pp.93-120). Santiago de Chile: Editorial Universitaria.

Avellar, S. \& Supplee, L. (2013). Effectiveness of home visiting in improving child health and reducing child maltreatment. Pediatrics, 132(2), 90-99. 
Berroeta Torres, H., Hatibovic Díaz, F. \& Asún Salazar, D. (2012). Psicología comunitaria: Prácticas en Valparaíso y visión disciplinar de los académicos nacionales. Polis, 11(31), 335-354.

Brofenbrenner, U. (1986). Ecology of the family as a context for human development: Research perspectives. Developmental Psychology, 22(6), 723-742.

Chile, Encuesta de Caracterización Socioeconómica Nacional [CASEN] (2013). Encuesta CASEN 2013: Módulo educación, síntesis de resultados. Centro de Estudios (MINIEDUC). Extraído del sitio Web del Ministerio de Desarrollo Social de Chile: http://observatorio. ministeriodesarrollosocial.gob.cl/documentos/Casen2013_Educacion.pdf.

Cornejo, R. \& Redondo, J. M. (2007). Variables y factores asociados al aprendizaje escolar: Una discusión desde la investigación actual. Revista Estudios Pedagógicos, 33(2), 155-175.

Cowan, G., Bobby, K., St. Roseman, P. \& Echandia, A. (2002). Evaluation report: The home visit project. U.S. Department of Education Office of Educational Research and Improvement. Recuperado el 26 de mayo 2017 de: http://files.eric.ed.gov/fulltext/ED466018.pdf.

Flick, U. (2012). Introducción a la investigación cualitativa. (3ª . Ed.). Madrid, España: Morata.

Franklin, C., Kim, J. \& Tripodi, S. (2009). A meta-analysis of published school social work practice studies 1980-2007. Research on Social Work Practice, 19(6), 667-677.

Fundación Paz Ciudadana. (2017). Proyecto sistematización experiencia planes integrales para barrios de alta complejidad (3er Informe: Principales aprendizajes, desafíos y recomendaciones del traspaso del modelo de intervención). Extraído del sitio Web de Fundación Paz Ciudadana: https://pazciudadana.cl/biblioteca/documentos/ sistematizacion-planes-integrales-para-barriosde-alta-complejidad/.

. (2018). Encuesta de victimización, inseguridad y percepción local en barrios de San Joaquín. Extraído del sitio Web de Fundación Paz Ciudadana: https://pazciudadana.cl/biblioteca/ciudad/ encuesta-de-victimizacion-inseguridad-y-percepcion-local-en-la-legua/.

Gatica, F. \& Alfaro, J. (2015). Las intervenciones psicosociales en establecimientos educacionales municipales vulnerables bajo el marco de la ley SEP (Tesis para optar a grado de magister en Gestión y Políticas Públicas). Universidad de Chile, Santiago, Chile.

García, J. \& Cohen, G. (2010) Social psychological perspective on educational intervention. In: E. Shafir (Ed.), The Behavioral Foundations of Policy. (pp. 329-347). New Jersey, EE. UU.: Princeton University Press.

Gastañaga, J. L. (2007) Construyendo posibilidades: intervención psicosocial en la escuela. Cuadernos de Trabajo Social, 20, 189-207

Gavilán, M., Quiles, C. \& Chá, T. (2005). Paradoja: Alta problemática psicosocial-baja intervención orientadora. Orientación y Sociedad, 5, 141-156.

Gómez, E. \& Haz, A. M. (2008). Intervención Familiar Preventiva en Programas Colaboradores Del SENAME: La Perspectiva Del Profesional. Psykhe, 17(2), 53-65.

Jadue, G. (2002) Factores psicológicos que predisponen al bajo rendimiento, al fracaso y a la deserción escolar. Revista Estudios de pedagogía, 28, 193-204.

Larraín, S. (2011). De objeto de protección a sujeto de derecho. En Cousiño, F. \& Foxley, A. M. (Ed.). Políticas públicas para la infancia. Santiago, Chile: UNESCO

Leal-Soto, F. (2005). Lo psicosocial en contextos educativos: consideraciones conceptuales y empíricas a partir de una experiencia en liceos de alta vulnerabilidad. Límite, Revista de Filosofía y Psicología, 12, 51-104.

Ley general de educación. Ley $\mathrm{N}^{\circ}$ 20.370. Biblioteca del congreso nacional de Chile, Santiago, Chile, 12 de septiembre de 2009.

Ley de subvención escolar preferencial. Ley $\mathrm{N}^{\circ}$ 20.248. Biblioteca del congreso nacional de Chile, Santiago, Chile, 25 de enero del 2008.

López, V., Carrasco, C., Morales, M., Ayala del Castillo, A., López, J. \& Karmy, M. (2011). Individualizando la violencia escolar: Análisis de prácticas discursivas en una escuela municipal de la región de Valparaíso. Psykhe, 20(2), 75-91. 
Martínez, M. (2006). La investigación cualitativa (síntesis conceptual). Revista de Investigación en Psicología, 9(1), 123-146.

Meyer, J., Mann, M. \& Becker, J. (2011) A five-year follow-up: Teachers' perceptions of the benefits of home visits for early elementary children. Early Childhood Education Journal, 39(3), 191196.

Ministerio de Salud [MINSAL]. (2008). Orientaciones técnicas: Visita domiciliaria integral para el desarrollo biopsicosocial de la infancia. Gobierno de Chile: Ministerio de Salud. Disponible en: www.crececontigo.cl.

[MINSAL]. (2013). Programa Nacional de Salud de la Infancia con Enfoque Integral. Gobierno de Chile: Ministerio de Salud. Disponible en: www.minsal.cl.

Office of Planning, Research and Evaluation [OPRE]. (2016). Home visiting evidence of effectiveness review. Executive summary, 85(a).

Razeto, A. (2016). Estrategias para promover la participación de los padres en la educación de sus hijos: el potencial de la visita domiciliaria. Revista Estudios Pedagógicos, 42(2), 449-462.

Santana, A. I. \& Farkas, C. (2007). Estrategias de autocuidado en equipos profesionales que trabajan en maltrato infantil. Psykhe, 16(1), 77-89.

Saracostti, M. (2013). Familia-Escuela Comunidad III: una alianza necesaria para un modelo de intervención biopsicosocial positivo. Santiago de Chile: Editorial Universitaria.

Saracostti, M. \& Santana, A. I. (2013). Corporación Municipal de Peñalolén. En: Saracostti, M. (Ed.). Familia-Escuela Comunidad I: una alianza necesaria para un modelo de intervención biopsicosocial positivo (pp. 93-120). Santiago de Chile: Editorial Universitaria.

Schodt, S. Parr, J. Araujo, M. C. \& Rubio-Codina, M. (2015). La medición de la calidad de los servicios de visita domiciliarias, una revisión de la literatura. $B D I$.

Siverio, A. M. (2011). La Contextualización del modelo de atención educativa no institucional cubano "Educa a tu Hijo" en países latinoamericanos. UNICEF y Organización de Estados Iberoamericanos (OEI).

Vásquez, A. (2015) Apoyo psicosocial para la promoción de habilidades para la vida: Una experiencia de intervención desde el rol del psicólogo social en el contexto educativo. Revista de Psicología GEPU, 6(1), 137-142.

Walsh, M., Madaus, G., Raczek, A., Dearing, E., Foley, C., An, Ch., Lee-St John, T. \& Beaton, A. (2014). A new model for student support in high-poverty urban elementary schools, effects on elementary and middle school academic outcomes. American Educational Research Journal, 51(4), 704-737.

Walsh, M., Lee-St John, T., Raczek, A. \& Foley, C. (2015). The long-term impact of systematic student support in elementary school: Reducing high school dropout. Society for Research on Educational Effectiveness (SREE). Conference Abstract Template.

Wasik, B. \& Bryant, D. (2001). Home visiting. Procedures for helping families. Thousand Oaks, CA: Sage.

Yager, D. \& Walton, G. (2011). Social-Psychological interventions in education: They're not magic. Review of Educational Research, 81(2), 267-301. 